\title{
Studies of vacuum photomultipliers at extremely low thresholds, photoelectron backscattering and photon detection efficiency
}

\section{Bayarto Lubsandorzhiev ${ }^{1}$}

Institute for Nuclear Research of the Russian Academy of Sciences

117312 Moscow, pr-t 60-letiya Oktyabrya 7a, Russia

E-mail:lubsand@rambler.ru

\section{Evgeny Vyatchin}

Institute for Nuclear Research of the Russian Academy of Sciences 117312 Moscow, pr-t 60-letiya Oktyabrya 7a, Russia

E-mail:lubsand@rambler.ru

\section{Sultim Lubsandorzhiev}

Institute for Nuclear Research of the Russian Academy of Sciences 117312 Moscow, pr-t 60-letiya Oktyabrya 7a, Russia

E-mail:lubsand@rambler.ru

\section{Andrei Sidorenkov}

Institute for Nuclear Research of the Russian Academy of Sciences 117312 Moscow, pr-t 60-letiya Oktyabrya 7a, Russia

E-mail:lubsand@rambler.ru

\section{Sergey Tsydenzhapov}

Institute for Nuclear Research of the Russian Academy of Sciences 117312 Moscow, pr-t 60-letiya Oktyabrya 7a, Russia

E-mail:lubsand@rambler.ru

We present results of studies of vacuum photomultipliers behavior at extremely low thresholds.

International Conference on New Photo-detectors

PhotoDet 2015

6-9 July 2015

Moscow, Troitsk, Russia

${ }^{1}$ Speaker

(C) Copyright owned by the author(s) under the terms of the Creative Commons 


\section{Introduction.}

Usually in case of registration of low intensity light, like in Cherenkov and air fluorescent light detection experiments as well as in laboratory studies of single photoelectron response of photomultipliers, photomultipliers are operated at thresholds in the range of 0.1-0.5 photoelectrons (p.e.). But what will happen if to decrease threshold further down? For many years experimental physicists have been plagued by a sharp rise of the number of pulses with low charges in the charge distribution of single photoelectron pulses when they have been trying to decrease threshold. This part of the spectrum has been attributed usually to the "noise" pulses ("dynode" or whatever noise) and erroneously discarded. In our previous works $[1,2]$ it was shown that the part of the spectrum due to pulses with low charge are explained by photoelectrons inelastically backscattered on the first dynode or other construction elements. This part of the spectrum is of crucially importance for measurements and understanding of absolute photon detection efficiency of classical vacuum photomultipliers.

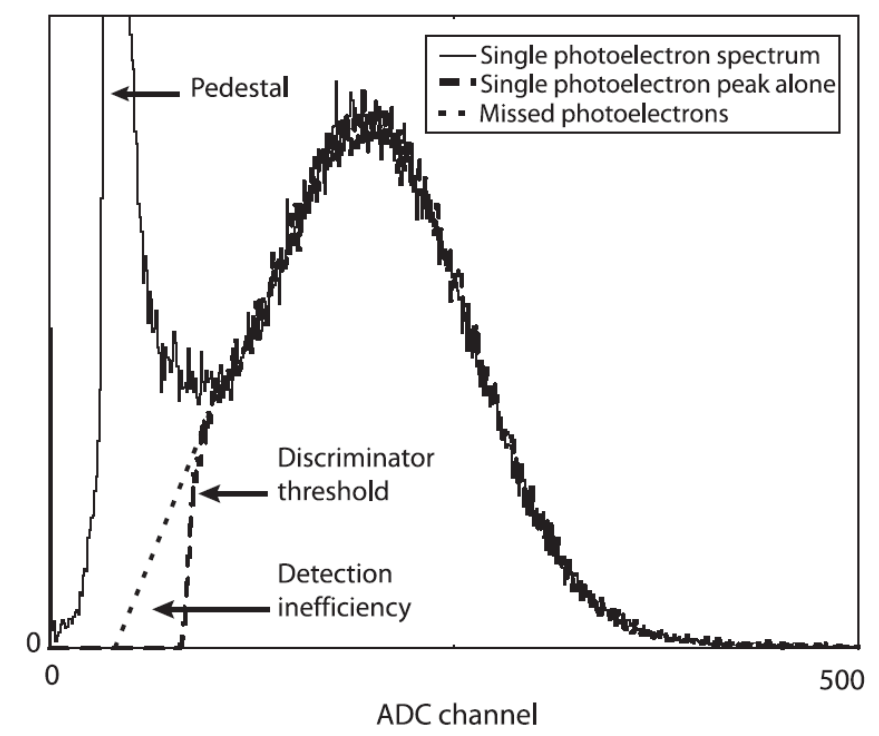

Fig. 1. Charge spectrum of single photoelectron pulses for Photonis XP2020Q PMT [3].

The most common mistake made often by experimenters is demonstrated in Fig. 1 quoted from [3]. The authors of the paper simply discarded left part of the measured single photoelectron spectrum approximating 1 p.e. peak to zero. After that they inferred the wrong value for efficiency of single photoelectron detection. This wrong value they used to get the final result of their studies.

The cause of such mistakes originates probably from the fact that usually experimenters work at relatively high thresholds corresponding to $0.25-0.5$ p.e. But what will happen if to decrease threshold to values less than 0.1 p.e. and even further down?

It should be noted here that it was pointed out in [4] on the necessity to take into account low amplitude part of single photoelectron spectrum. 


\section{Set-up and measurements results.}

Trying to decrease threshold one inevitably encounters with many problems, the most formidable of them is an electronic pick-up which threatens to destroy all measurements. To avoid such problems we developed and built a dedicated set-up which incorporated metallic box surrounded by well grounded Faraday cage, low noise fast transimpedance preamplifier based on HEWLETT PACKARD INA-03184 chip, coincidence techniques etc. PMT under study was fixed in the metallic box. We studied a number of PMTs of different types and sizes - from half inch to 20 inch PMTs.

Decreasing threshold working with some photomultipliers we managed to set a record threshold of as low as 0.002 p.e.. At such extremely low threshold beside main single photoelectrons from photocathode we detected photoelectrons produced by direct photoemission at the first and even second dynodes. In Fig. 2 the charge distribution of single photoelectron pulses of R1463 Hamamatsu PMT is presented. The PMT is 0.5 " photomultiplier and its gain was $\sim 10^{7}$. At the lowest threshold we registered two small peaks in the most left part of the spectrum. To understand the origin of those two peaks the PMT's photocathode and first dynode were consecutively switched off and charge spectrum was measured with the same intensity of lightr pulses. These procedures proved that the two peaks are due to the direct photoeffect on the first and second dynodes. The spectrum in green in Fig. 2 was measured with the PMT's photocathode switched off. So, carefully measuring this effect it is possible to subtract events due to the photoeffect on the first and second dynodes from the PMT's single photoelectron charge spectrum. The spectrum after subtraction is shown in Fig. 3.

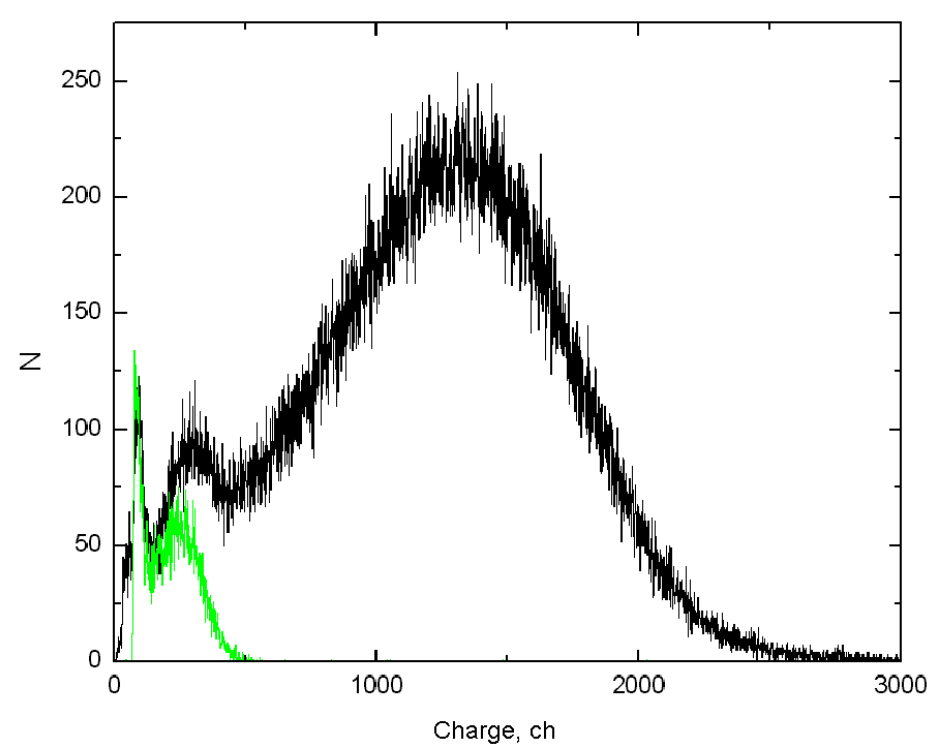

Fig. 2. Charge spectrum of single photoelectron pulses for R1463 PMT at threshold corresponding to $\sim 0.002$ p.e..

It is important that simultaneous photoelectron transit time measurements confirm conclusions on direct photoeffect events on the first and second dynodes of the PMT [2]. 


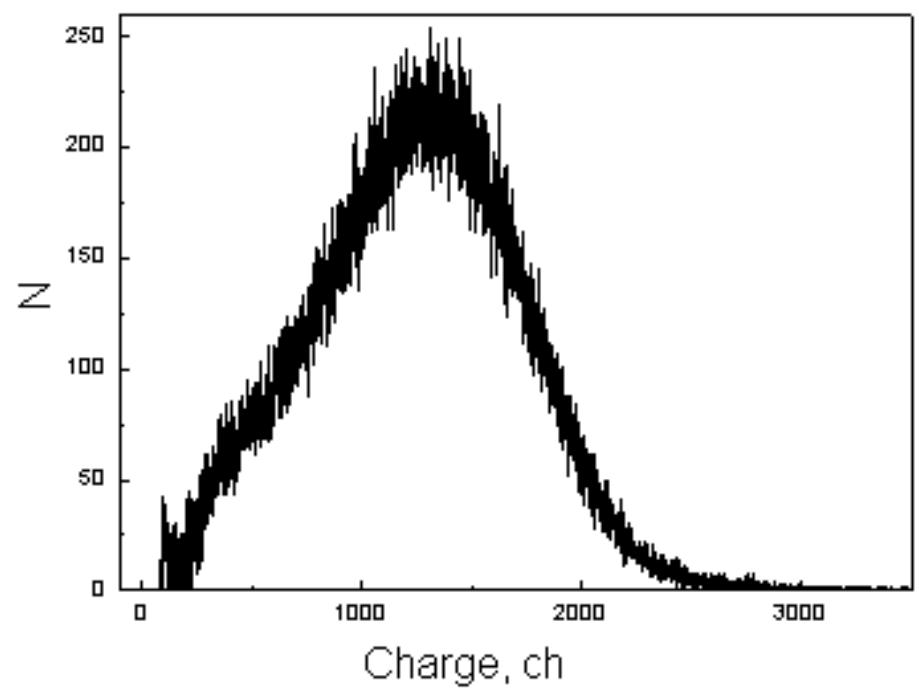

Fig. 3. Charge spectrum of single photoelectron pulses for R1463 PMT at threshold corresponding to $\sim 0.002$ p.e. after subtraction of events due to the direct photoeffect on the first and second dynodes.

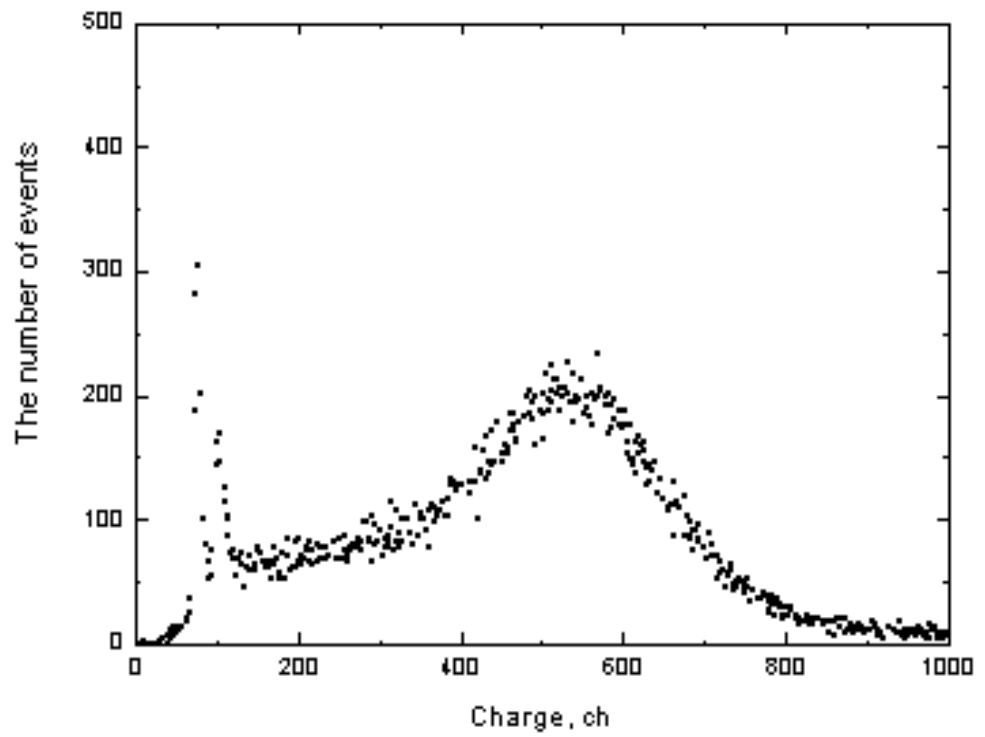

Fig. 4. Charge spectrum of single photoelectron pulses for 13" R8055 PMT at threshold corresponding to $\sim 0.002$ p.e..

Large sensitive area PMTs show the same behavior at extremely low thresholds. Although they set additional problems connected with their sizes. In Fig. 4 the charge spectrum of single photoelectron pulses for 13" R8055 photomultiplier at $10^{7}$ gain is shown. The lowest threshold reached with this PMT is practically the same value of $\sim 0.002$ p.e. as in case of R1463. In the left part of spectrum around channel number $\sim 100$ there is also very narrow peak due to the 
direct photoeffect on the first dynode. It is interesting that there is no peak connected with the second dynode. Probably in R8055 the second dynode is much better optically hidden in contrary to R1463.

Although we reached such low threshold with photomultipliers of different types and sizes, from $1 \mathrm{~cm}$ to half a meter in photocathode's diameter, unfortunately not every photomultiplier is able to operate at such threshold (here it means a combined effect of threshold and PMT gain). On the other hand photomultipliers able to work at such extremely low thresholds are in general prone to be more stable and have better single photoelectron response. So it is possible to put forward a new criterion for photomultipliers quality - the ability to work at thresholds lower than 0.01 p.e..

\section{Conclusion.}

It was demonstrated that there are PMTs of different sizes and types which can operate at thresholds as low as 0.002 p.e.. Such PMTs are generally prone to be more stable in wide range of operating voltages allowing to set a new criterion of PMT quality. Operation of vacuum photomultipliers at extremely low thresholds highlights fundamental problem of photon detection - absolute photon detection efficiency which is of utmost importance for physics experiments using vacuum photomultipliers. The developed method to work with extremely low threshold will allow to trace the fate of each photoelectron produced on the photocathode and will improve the accuracy of photon detection efficiency with vacuum photomultipliers.

\section{Acknowledgements.}

The work was supported by the Russian Foundation for Basic Research (Grants 13-029244-ASPERA-a and 13-03-12451-ofi-m). The author is indebted very much to Dr. V.Ch. Lubsandorzhieva for careful reading of the manuscript, invaluable discussions and many useful corrections.

\section{References}

[1] B.K. Lubsandorzhiev et al. Nuclear Instruments and Methods A. 2000. V.442. P.452.

[2] B.K. Lubsandorzhiev et al. Nuclear Instruments and Methods A. 2006. V.567. P.12.

[3] G. Lefeuvre et al. Nuclear Instruments and Methods A. 2007. V.578. P.78.

[4] R. Mirzoyan, E. Lorenz. Proc. of $25^{\text {th }}$ ICRC. Durban South Africa. 1997. P.265. 